\title{
Neutron spin rotation effect at Laue diffraction in a weakly deformed and nonabsorbing crystal with no center of symmetry
}

\author{
Vladimir Voronin $^{1,2,3, a}$, Valery Fedorov ${ }^{1,2,3}$, Sergey Semenikhin ${ }^{1,2}$, and Yaroslav Berdnikov ${ }^{2}$ \\ ${ }^{1}$ Petersburg Nuclear Physics Institute, National Research Center Kurchatov Institute, Gatchina 188300, Russia \\ ${ }^{2}$ Peter the Great St. Petersburg Polytechnic University, St. Petersburg 195251, Russia \\ ${ }^{3}$ St. Petersburg State University, St. Petersburg 199034, Russia
}

\begin{abstract}
The effect of the neutron spin rotation at Laue diffraction in a weakly deformed noncentrosymmetric and transparent for the neutrons crystal has been theoretically described and experimentally investigated. This effect arises in the deformed crystal because of the curvature of the neutron trajectory in the crystal. A certain type of deformation leads to the escape outside the crystal of one of the two neutron waves excited at Laue diffraction. This two waves propagate in the crystal without a center of symmetry in electric fields with the opposite sign. In this case the spin of the remaining neutron wave will be rotating relative to the original direction due to the interaction of the magnetic moment of the moving neutron with the crystal's intracrystalline electric field. In a perfect undeformed crystal such spin rotation effect is absent. There is only a depolarization of the beam since both waves in opposite electric fields are present with the same amplitudes.

A technique for controlled deformation of a perfect single crystal by creating a temperature gradient has been developed. Thus a new possibility to measure the electric fields which act on the neutron in noncentrosymmetric crystals has been realized. There also appeared a way to control these fields in experiments on the study of the neutron fundamental properties.
\end{abstract}

\section{Introduction}

It follows from the dynamic theory of diffraction [1] that the propagation of a neutron in a crystal in directions, close to the Bragg's ones for a certain system of crystallographic planes, can be described by two types of Bloch waves $\psi^{(1)}$ and $\psi^{(2)}$. These waves are formed in the crystal as a result of the interaction of a neutron with the periodic nuclear potential of a given plane system that is characterized by a reciprocal lattice vector $\mathbf{g}$ directed perpendicular to the planes and equal in absolute value $|\mathbf{g}| \equiv \mathrm{g}=2 \pi / d$, where $d$ is the interplanar distance. The corresponding harmonic of the periodic nuclear potential has the form:

$$
V_{g}^{N}(\mathbf{r})=2 V_{g}^{N} \cos \mathbf{g r} .
$$

The amplitude of the harmonic $V_{g}^{N}$ is determined by the structural amplitude of nuclear neutron scattering by a crystal cell $F_{g}^{N}$ :

$$
V_{g}^{N}=\frac{2 \pi \hbar^{2}}{m} N_{c} F_{g}^{N} ; F_{g}^{N}=\sum_{i} e^{-W_{i g}} f_{i}^{N}(\mathbf{g}) e^{i \mathbf{g r}_{i}} .
$$

Here $m$ is the neutron mass, $N_{c}$ is number of elementary cells in a unit volume of the crystal, $i$ is the index of the atom in the unit cell, $\mathbf{r}_{i}$ is the position of atomic nucleus in the unit cell, $f_{i}^{N}(\mathbf{g})$ is the scattering amplitude by the $i$-th nucleus of a cell with momentum transfer $\hbar \mathbf{g}, W_{i g}$ is a Debye-Waller factor. Note that the structural forward scattering amplitude $(\mathbf{g}=0)$ determines the average nuclear potential of the crystal $V_{0}^{N}$ and its average refractive index.

The waves $\psi^{(1)}$ and $\psi^{(2)}$ are two orthogonal superpositions of the direct wave with the wave vector $\mathbf{k}$ and reflected by crystallographic planes with the wave vector $\mathbf{k}+\mathbf{g}$ :

$$
\begin{aligned}
& \psi^{(1)}(\mathbf{r})=\cos \gamma e^{i \mathbf{k}^{(1)} \mathbf{r}}+\sin \gamma e^{i\left(\mathbf{k}^{(1)}+\mathbf{g}\right) \mathbf{r}}, \\
& \psi^{(2)}(\mathbf{r})=-\sin \gamma e^{i \mathbf{k}^{(2)} \mathbf{r}}+\cos \gamma e^{i\left(\mathbf{k}^{(2)}+\mathbf{g}\right) \mathbf{r}} .
\end{aligned}
$$

Here

$$
\tan 2 \gamma=\frac{U_{g}^{N}}{\Delta_{g}} \equiv \frac{1}{w_{g}},
$$

where

$$
\begin{aligned}
U_{g}^{N} & =\frac{2 m V_{g}^{N}}{\hbar^{2}}, \Delta_{g}=\frac{\left[(\mathbf{k}+\mathbf{g})^{2}-k^{2}\right]}{2} \\
& \equiv \frac{\left(k_{g}^{2}-k^{2}\right)}{2}=\frac{\left(2 \mathbf{k g}-g^{2}\right)}{2}
\end{aligned}
$$

is having dimension and $w_{g}$ is dimensionless deviation parameter from the Bragg condition. Below we will also use the dimensionless parameter

$$
\alpha_{g}=\frac{\left[(\mathbf{k}+\mathbf{g})^{2}-k^{2}\right]}{2 k^{2}}=\frac{\Delta_{g}}{k^{2}}=w_{g} \frac{U_{g}^{N}}{k^{2}} .
$$

The wave vectors $\mathbf{k}^{(1)}$ and $\mathbf{k}^{(2)}$ belong to different branches of the dispersion surface whose equation is

$$
\left(\mathbf{k}^{(1,2)}\right)^{2}=K^{2}-\Delta_{g} \pm \sqrt{\Delta_{g}^{2}+\left(U_{g}^{N}\right)^{2}} .
$$

(c) The Authors, published by EDP Sciences. This is an Open Access article distributed under the terms of the Creative Commons Attribution License 4.0 (http://creativecommons.org/licenses/by/4.0/). 


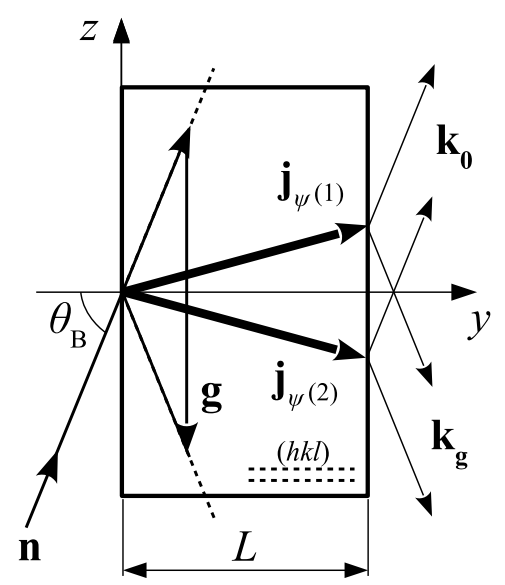

Figure 1. Symmetric case of Laue diffraction in a bounded nondeformed crystal. Neutrons $\mathbf{n}$ fall on the crystal at an angle different from the Bragg angle $\theta_{\mathrm{B}}$ within width of the Bragg (Darwin). $\mathbf{j}_{\psi^{(1)}}$ and $\mathbf{j}_{\psi^{(2)}}$ are vectors of neutron current densities of two Bloch waves, $\mathbf{g}$ is the direction of the reciprocal lattice vector.

Here $K$ is the value of the wave vector of a neutron in crystal taking into account the average refractive index of the crystal $K^{2}=k_{e}^{2}\left(1-V_{0}^{N}\right), k_{e}$ is the wave vector of incident neutron in vacuum.

For the value $\cos ^{2} \gamma$ we have

$$
\begin{aligned}
\cos ^{2} \gamma & =\frac{1}{2}\left[1+\frac{\Delta_{g}}{\sqrt{\Delta_{g}^{2}+\left(U_{g}^{N}\right)^{2}}}\right] \\
& =\frac{1}{2}\left[1+\frac{w_{g}}{\sqrt{1+w_{g}^{2}}}\right]
\end{aligned}
$$

The densities of the neutron probability currents in the branches witch averaged over rapid oscillations with a period $d$ are equal to

$$
\begin{aligned}
\mathbf{j}_{\psi^{(1)}} & =\frac{\hbar}{m}\left[\mathbf{k}^{(1)} \cos ^{2} \gamma+\left(\mathbf{k}^{(1)}+\mathbf{g}\right) \sin ^{2} \gamma\right] \\
& =\frac{\hbar}{m}\left[\left(\mathbf{k}^{(1)}+\frac{\mathbf{g}}{2}\right)-\frac{\mathbf{g}}{2} \frac{w_{g}}{\sqrt{1+w_{g}^{2}}}\right], \\
\mathbf{j}_{\psi^{(2)}} & =\frac{\hbar}{m}\left[\mathbf{k}^{(2)} \sin ^{2} \gamma+\left(\mathbf{k}^{(2)}+\mathbf{g}\right) \cos ^{2} \gamma\right] \\
& =\frac{\hbar}{m}\left[\left(\mathbf{k}^{(2)}+\frac{\mathbf{g}}{2}\right)+\frac{\mathbf{g}}{2} \frac{w_{g}}{\sqrt{1+w_{g}^{2}}}\right] .
\end{aligned}
$$

Under exact Bragg condition $\left(w_{g}=0\right)$ the wave $\psi^{(1)}$ and $\psi^{(2)}$ are symmetric and antisymmetric combinations of the direct and reflected waves. Their propagation takes place along the crystallographic planes (in the direction $\mathbf{k}_{\|}=\mathbf{k}+\mathbf{g} / 2$, see Fig. 1) and the neutrons in the $\psi^{(1)}$ state are concentrated mainly on the nuclear planes (by the "nuclear" planes we mean the positions of the maxima of the nuclear potential) but in the state $\psi^{(2)}$ between them. For this reason, neutrons in states $\psi^{(1)}$ and $\psi^{(2)}$ move in different potentials and have slightly different kinetic energies (i.e. different values of wave vectors). The deviation from the Bragg condition leads to changes in the direction of current densities $\mathbf{j}_{\psi^{(1)}}$ and $\mathbf{j}_{\psi^{(2)}}$ in opposite directions.

For the wave function inside the crystal in the symmetric Laue diffraction scheme (when the entrance face of the crystal is perpendicular to the reflecting planes) from the boundary conditions it follows:

$$
\psi(\mathbf{r})=\cos \gamma \psi^{(1)}(\mathbf{r})+\sin \gamma \psi^{(2)}(\mathbf{r}) .
$$

So, for small deviations from the Bragg condition $\left(w_{g} \ll 1\right)$ both states are excited with practically the same probability. However, at Bragg angles $\theta_{\mathrm{B}}$ close to $90^{\circ}$ (i.e. when $\left.\mathbf{k}_{\|} \ll \mathbf{g} / 2, \tan \theta_{\mathrm{B}}=g / 2 k_{\|}\right)$the directions of the currents can change very much (see Fig. 1):

$$
\mathbf{j}_{\psi^{(1,2)}} \approx \frac{\hbar}{m}\left[\mathbf{k}_{\|}^{(1,2)} \pm \frac{\mathbf{g}}{2} w_{g}\right]
$$

\section{Diffraction in noncentrosymmetric crystals}

In noncentrosymmetric crystals, for certain systems of crystallographic planes, the positions of the maxima of the electric potential can be shifted relative to the maxima of the nuclear potential:

$$
V_{g}^{E}(\mathbf{r})=2 V_{g}^{E} \cos \left(\mathbf{g r}+\phi_{g}\right) .
$$

Therefore neutrons in the states $\psi^{(1)}$ and $\psi^{(2)}$ are in strong $\sim 10^{8}-10^{9} \mathrm{~V} / \mathrm{cm}$ interplanar electric fields of the opposite sign $\pm \mathbf{E}_{g}$ directed along the vector $\mathbf{g}$ [2-4].

$$
\begin{aligned}
\left\langle\psi^{(1)}\left|\mathbf{E}_{g}(\mathbf{r})\right| \psi^{(1)}\right\rangle & =-\left\langle\psi^{(2)}\left|\mathbf{E}_{g}(\mathbf{r})\right| \psi^{(2)}\right\rangle \\
& \equiv \mathbf{E}_{g}=V_{g}^{E} \mathbf{g} \sin \phi_{g}
\end{aligned}
$$

where

$$
\mathbf{E}_{g}(\mathbf{r})=-\nabla V_{g}^{E}(\mathbf{r})=2 V_{g}^{E} \mathbf{g} \sin \left(\mathbf{g r}+\phi_{g}\right) .
$$

In the moving frame of reference associated with the neutron, neutrons in the different states will be affected by "Schwinger" magnetic fields with opposite signs $\mathbf{H}_{g}^{S}=$ $\pm\left[\mathbf{E}_{g} \times \mathbf{v}\right] / c$, where $\mathbf{v}$ is the neutron velocity, $c$ is the speed of light. Consequently, the spin (magnetic moment) of the neutron in the states $\psi^{(1)}$ and $\psi^{(2)}$ will precess around $\mathbf{H}_{g}^{S}$ in opposite directions. As a result, at Laue diffraction with the spin initially oriented perpendicular to the "Schwinger" magnetic field (i.e. in the diffraction plane), after passes through the crystal the spin of one half neutrons will turn by the angle $\phi_{S}$, and the other half by the angle $-\phi_{S}$ :

$$
\phi_{S}=\frac{4 \mu H_{g}^{S} L}{\hbar v_{\|}}=2 \mu_{n} \frac{e E_{g} L}{m_{p} c^{2}},
$$

where $v_{\|}$is the neutron speed in the crystal along the crystallographic planes, $\mu_{n}=-1.9$ is the magnetic moment of the neutron in units of nuclear magneton, $L$ is the thickness of the crystal, $m_{p}$ is the mass of the proton, $c$ is the speed of light. This leads to the depolarization of diffracted beams [5,6] (both direct and reflected, see Fig. 2).

When the initial polarization is perpendicular to the vector $\mathbf{H}_{g}^{S}$ and a certain thickness of the crystal $L_{0}$, the spin of neutrons is rotated by an angle of 90 degrees in opposite directions, thus there will be a complete depolarization 


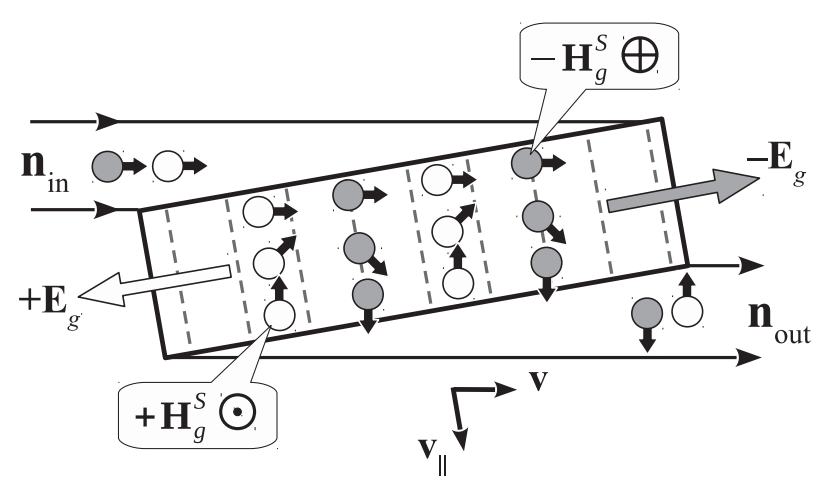

Figure 2. Spin behavior of neutrons in a perfect crystal without center of symmetry at Laue diffraction. Due to the interaction of the magnetic moment of the neutron with the Schwinger magnetic field, the spin of the neutron for two Bloch states rotates in opposite directions. In figure: $\mathbf{v}_{\|}-$the neutrons velocity direction along the crystallographic planes, $\mathbf{n}_{\text {in }}, \mathbf{n}_{\text {out }}-$ direction of the incident and diffracted neutrons.

of neutrons passed through the crystal. For the plane system (110) of a $\alpha$-quartz crystal $L_{0} \approx 3.5 \mathrm{~cm}$ [7]. The deviation from the Bragg condition leads to a decrease of the electric field acting on the neutron and, consequently, to an increase of the thickness of the crystal. If the neutrons are polarized along the vector $\mathbf{H}_{g}^{S}$, their polarization will not be going to change after passing through the crystal.

With the exact equality of the amplitudes of the two Bloch waves (which occurs only under the exact Bragg condition), the mean field acting on the neutron in the crystal is zero, so the spin rotation effect is absent. However, a deviation from the Bragg condition leads to an imbalance of these amplitudes. In this case, along with depolarization, neutron spin rotation can also occur. As a result due to the small gradient of interplanar distance, the deformation of the crystal can violate the equality of the amplitudes of waves $\psi^{(1)}$ and $\psi^{(2)}$, respectively, to cause the effect of neutron spin rotation at Laue diffraction in a nonabsorbing crystal. The possibility of spin rotation in the absorbing crystal due to different absorption in the crystal of $\psi^{(1)}$ and $\psi^{(2)}$ waves (Bormann effect) was discussed in [8].

The neutron spin rotation effect due to the Schwinger interaction at Laue diffraction in a virtually nonabsorbing non-centrosymmetric crystal have been discovered first in a test experiment to search for the electric dipole moment (EDM) of the neutron by the diffraction method [9]. In that work, the spin polarization component of a neutron parallel to $\mathbf{H}_{g}^{S}$ have been measured, which appears as a result of an additional spin rotation of a neutron due to the interaction of neutron EDM with the electric field of a noncentrosymmetric crystal. The spin rotation of the neutron due to the Schwinger interaction could lead to a false effect, which is necessary to study to exclude systematic errors in experiments of this kind, see [10].

In this work the neutron spin rotation effect due to the Schwinger interaction depending on the deformation parameter of the crystal is studied in detail. This effect can be smoothly changed, for example, by heating and cooling different areas of the crystal. Preliminary results were published in [11].

The effect occurs if the parameter of deviation from the Bragg condition changes during the diffraction. It can be caused both by the change of the interplanar distance and the neutron energy (wavelength), for example, in the gravity field or other external force. In case of deviation from the exact Bragg condition, in addition to changes in the amplitudes of Bloch waves of different types, the balance of direct and reflected waves in each of the states $\psi^{(1)}$ and $\psi^{(2)}$ is also disturbed. As a result, the propagation of neutrons in these states will not occur along the crystallographic planes, but between the directions of the direct and reflected waves. At diffraction angles close to $90^{\circ}$ and small deviations from the exact Bragg angle, the amplitudes of the states $\psi^{(1)}$ and $\psi^{(2)}$ vary slightly but the direction of the neutron current density in these states can vary significantly. In general case, a small deviation $\Delta \lambda_{\mathrm{B}}\left(\Delta \lambda_{\mathrm{B}} / \lambda \sim 10^{-5}\right)$ of the wavelength of incident neutrons within the Darwin width or neutron direction from the Bragg conditions will change the direction inside the crystal within the range from the direction of the initial wave vector $\mathbf{k}$ to the beam direction which reflected by crystal planes $\mathbf{k}+\mathbf{g}$. In this terminology, Bragg's condition corresponds to equality $|\mathbf{k}|=|\mathbf{k}+\mathbf{g}|$.

\section{Neutron diffraction in a deformed crystal}

The neutron behavior in a deformed crystal can be described by using "Kato trajectories" [12], which are curves tangent to which are directed along the current density at each point of the trajectory, see Fig. 1. The physical meaning of these trajectories is that they describe the motion in a crystal of a two-wave packet of size along the entrance face exceeding $\xi_{g} \cdot \tan \theta_{B}$, where $\xi_{g}=$ $2 \pi /\left|\mathbf{k}^{(\mathbf{2})}-\mathbf{k}^{(\mathbf{1})}\right|$ is the extinction length.

In an undeformed crystal "Kato trajectory" are straight lines. The curvature of the trajectories in the crystal is determined by the degree of deformation of the crystal, which can be described by the "Kato force". So the "Kato trajectory" of neutron in the crystal at small deformations will be described by the equation [2]:

$$
\frac{\partial^{2} z}{\partial y^{2}}= \pm \frac{c_{0}}{m_{0}} f_{k}(y, z)
$$

where $c_{0}=\tan \theta_{\mathrm{B}}, m_{0} \equiv 2 d F_{g} / V_{c}$ is the "Kato mass", $F_{g}$ is the structural scattering amplitude of the neutron by the crystal cell, $v_{c}$ is volume of the crystal cell, $d$ is interplanar distance, and $f_{k}(y, z)$ is "Kato force":

$$
f_{k}(y, z)=\frac{k_{0}}{4 \cos \theta_{\mathrm{B}}}\left(\frac{\partial}{\partial z}+\frac{1}{c_{0}} \frac{\partial}{\partial y}\right) \alpha(y, z),
$$

where $k_{0}$ is the value of the neutron wave vector in the crystal, $\theta_{\mathrm{B}}$ is the Bragg angle. The parameter $\alpha(y, z)$ is deviation from the exact Bragg conditions:

$$
\alpha(y, z)=\frac{\left|\mathbf{k}_{0}+\mathbf{g}\right|^{2}-\mathbf{k}_{0}^{2}}{2 k_{0}^{2}}=\frac{\mathrm{g}^{2}+2\left(\mathbf{k}_{0} \mathbf{g}\right)}{2 k_{0}^{2}} .
$$

Changing this parameter in the crystal can be related both to the deformation of the crystal (i.e., to the change of the vector $\mathbf{g}$ ) and to the change of the moving direction of the neutron or its wavelength, for example, under the influence of an external force. Thus, in the case of a constant gradient 
of the interplanar distance, the neutron trajectories in the crystal will be described by the equation:

$$
\frac{\partial^{2} z}{\partial y^{2}}= \pm \frac{c_{0}^{2}}{m_{0}} \pi g \zeta,
$$

where $g=2 \pi / d$ is the value of the reciprocal lattice vector, $\zeta$ is the parameter characterizing the crystal linear deformation $\left(d=d_{0}(1+\zeta z)\right.$, where $d_{0}$ is the interplanar distance without deformation). The sign \pm in (19) corresponds to two different Bloch waves excited in the crystal.

For the case of quadratic deformation, i.e. $d=d_{0}(1+$ $\left.\xi z^{2}\right)$, the neutron trajectory will be determined by force:

$$
f_{k}=c_{0} \frac{2 \pi \xi z}{d},
$$

where $\xi$ is a parameter of quadratic deformation.

As a result, it turns out that for neutrons in one state, the Kato forces are directed to the center of the crystal $(z=0)$, and for the other state from the center (see (19). Thus, waves of one type will be focused, and the second will be defocused. Because of this, there will be a difference in the intensities of these waves at the exit face of the crystal. Moreover, if we change the sign of the deformation parameter, the waves will swap places. In a non-centrosymmetric crystal, these two waves will be in opposite electric (and, accordingly, Schwinger magnetic) fields. The spins of the neutron different waves will rotate in opposite directions, and after the passage through a quartz crystal with a thickness of $l_{0}=3.5 \mathrm{~cm}$ for the plane (110) will be directed oppositely. So, a change the crystal deformation parameter should lead to a change in the spin state of the diffracted neutron beam passed through the crystal.

It is easy to see that the right-hand side of Eq. (19) is proportional to $\tan ^{2} \theta_{\mathrm{B}}$. This value can reach values $\sim\left(10^{2}-10^{3}\right)$ when $\theta_{\mathrm{B}} \sim\left(84^{\circ}-88^{\circ}\right)$. Thus, the effect of small deformations on the neutron trajectory can be increased by $2-3$ orders of magnitude in comparison with conventional diffraction angles $\left(\theta_{\mathrm{B}} \sim 45^{\circ}\right)$.

Calculated neutron trajectories in a crystal deformed by temperature gradient $\Delta T^{0}=10^{-3} \mathrm{~K} / \mathrm{cm}$ shown in Fig. 3.

The calculation corresponds to the real size of the crystal $140 \times 35 \mathrm{~mm}^{2}$. The figure shows that even due to such small deformations, neutron fluxes for two Bloch waves diverge by several centimeters in the middle of the crystal. These calculations are in good agreement with experimental data.

\section{Experimental setup}

Experimental setup was mounted on the WWR-M reactor in Gatchina. The measurements were carried out on a direct diffracted neutron beam. The initial neutron flux at the experimental beam position was about $6 \cdot 10^{5} 1 /\left(\mathrm{cm}^{2}\right.$. $\AA$.s) for working wavelength $\lambda \sim 4.9 \AA$. A schematic diagram of the installation is shown in Fig. 4.

The unpolarized neutron beam (1) passes through a polarizer (2), where the initial polarization of the beam is directed along $x$-axis perpendicular to the plane of the figure. The value of the initial polarization of the beam

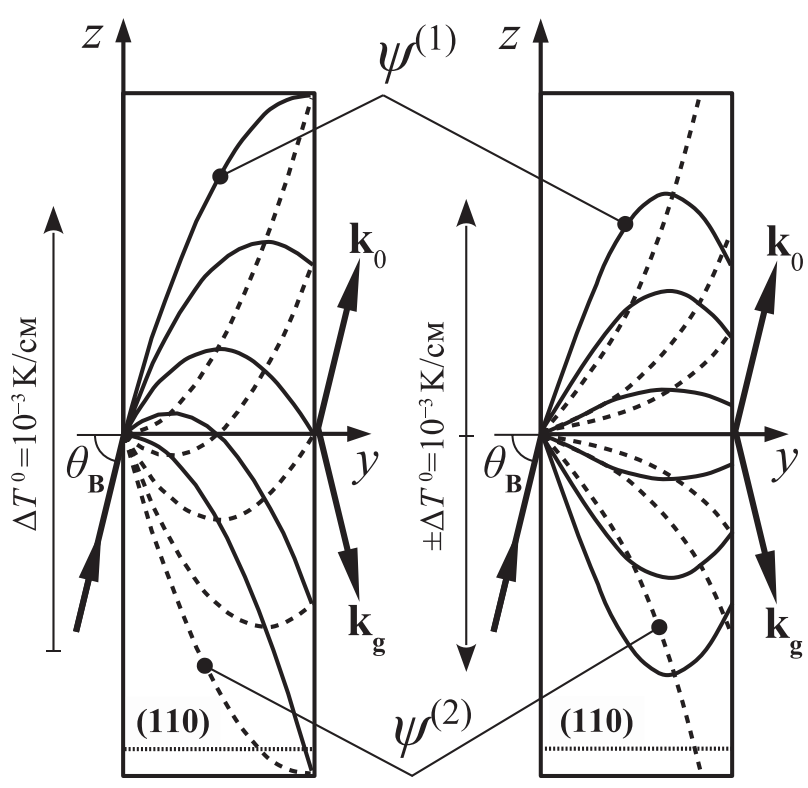

Figure 3. Calculation of the neutron trajectories inside the deformed by the temperature gradient $\Delta T^{0}=10^{-3} \mathrm{~K} / \mathrm{cm}$ along $z$ axis crystal of quartz (diffraction plane (110), the Bragg angle $\left.\theta_{\mathrm{B}}=86^{\circ}\right)$. The image on the left corresponds to a linear gradient along the $z$ axis, $d=d_{0}(1+\zeta z)$. The image on the right gradient along the $z$ axis from the center of the crystal to the top and bottom edges, $d=d_{0}\left(1+\xi z^{2}\right)$.

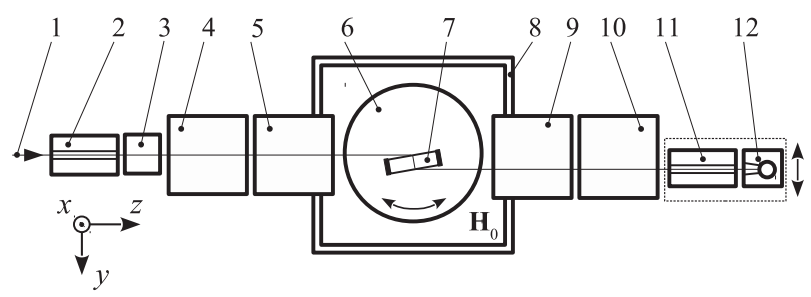

Figure 4. Scheme of the setup: 1 - neutron beam; 2,11 polarizing mirror-like multi-gap neutron guides (polarizer and analyzer); 3 - $\mathrm{BeO}$ neutron filter $(120 \mathrm{~mm}) ; 4,10$ - coils of guiding magnetic field $H \sim 4 \mathrm{Gs} ; 5,9$ are spin-rotating threecoordinate coils; 6 - turning table; 7 - the crystal unit; 8 magnetic shield; 12 - neutron detector with shield.

was $P_{0}=85 \%$. We used the polycrystalline BeO filter (the $120 \mathrm{~mm}$ thick) transmitting neutrons with the wavelength $\lambda>4.7 \AA$ to reduce the background from neutrons that can reach the detector after reflections from other planes of the crystal and from the working plane in higher diffraction orders.

Further, the beam, passing through a coil with guiding magnetic field (4) and an orienting spin-rotating threecoordinate coil (5), came on the crystal unit (7), which was located on the turntable (6) inside the double-layer magnetic shield (8). The turntable allows to rotate the crystal unit in the horizontal plane by $360^{\circ}$. The field inside the magnetic screen was $\mathrm{H}_{0}<0.01 \mathrm{Gs}$. The direction and value of diffracted neutron beam polarization was analyzed by the system of $3 \mathrm{D}$ polarization analysis consisting of spin-rotating three-coordinate coil (9), guiding field (10) and mirror multi-slit neutron guide analyzer (11). The ${ }^{3} \mathrm{He}$ detector (12) located on the moving platform was used for neutron registration. 


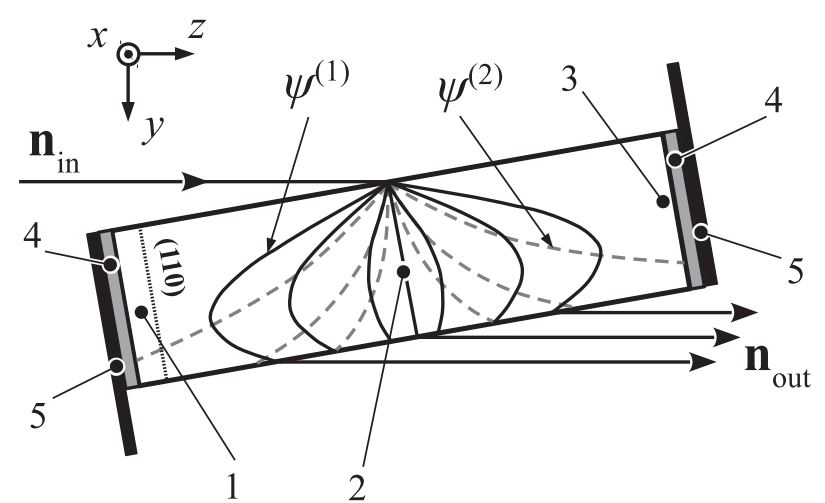

Figure 5. Scheme of the crystal unit: 1,2, 3 - points of the temperature measurement; 4 - Peltier elements, 5 - neutron absorbers; $\mathbf{n}_{\text {in }}, \mathbf{n}_{\text {out }}-$ directions of the incident and diffracted neutrons, respectively. Inside the crystal, the solid and dotted lines are the Kato trajectories waves $\psi^{(1)}$ and $\psi^{(2)}$.

The crystal size of quartz is $140 \times 35 \times 140 \mathrm{~mm}^{3}$. Working reflection plane is (110) with the interplanar distance $d=2.456 \AA$. The width of the beam collimation at the input face of the crystal is about $5 \mathrm{~cm}$. Thus, contribution to the diffracted beam (Fig. 5) will come from neutrons whose Kato trajectories start from this region. For example, with such collimation at the crystal and the working angle $82^{\circ}$, the counting rate on the detector (without background intensity) averaged about 0.6 neutron per second. The coefficient of quartz thermal expansion in the direction of reciprocal lattice vector for this plane is $1.3 \cdot 10^{-5} \mathrm{~K}^{-1}$.

Neutron absorbers and Peltier elements placed over the entire ends of the crystal (Fig. 5) create a required temperature distribution in the crystal. The ends of the crystal can be heated or cooled by changing the direction of the current in the elements. Calculations with the COMSOL software package showed that the temperature distribution over the $z$ axis inside the crystal is quadratic with a high accuracy under the experimental conditions and natural convection of air at simultaneous heating of the ends. The distribution is linear at the heating of one end and cooling of the other end. The temperature during the experiment was controlled by three Pt100 sensors at the ends and center of the crystal.

The electric field of the crystal $\pm \mathbf{E}_{g}$ was directed along the reciprocal lattice vector (see Fig. 2) and the Schwinger magnetic field $\pm \mathbf{H}_{g}^{S}$ along the axis $x$, respectively.

The external magnetic field on the crystal was $\mathbf{H}_{0} \ll$ $\mathbf{H}_{g}^{S}$, so they can be neglected. To study the spin rotation effect inside the crystal, vector of initial polarization was directed along the axis of the beam $z$, i.e. perpendicularly to the Schwinger magnetic field $\mathbf{H}_{g}^{S}$, and the polarization components along the axes $P_{z}$ and $P_{y}$ were measured.

The mechanism for the appearance of polarization is illustrated in Fig. 6. Here $\mathbf{N}_{i}$ and $\mathbf{N}_{f}$ are the initial and final polarization, respectively. The final polarization along $x$ axis is determined by the rotation of the neutron spin by an angle $\pm \phi_{S}$ (see Fig. 6 a)). While the polarization in the $y$ direction arises from the inequality of the intensities of two Bloch waves $\psi^{(1)}$ and $\psi^{(2)}$ at the crystal exit face caused by deformation (focusing of one Bloch wave and defocusing of another wave).

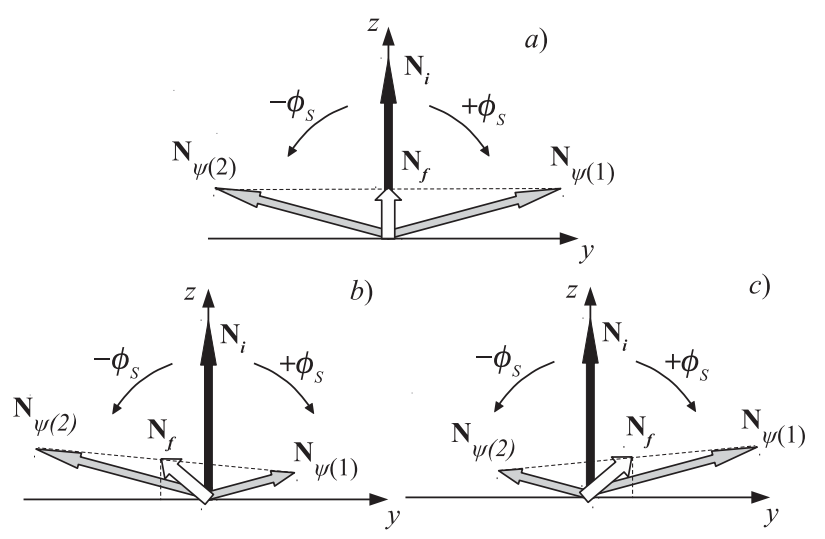

Figure 6. The mechanism of the appearance of the polarization component $P_{x}: \mathbf{N}_{i}$ and $\mathbf{N}_{f}$ are the initial and final polarization; $\mathbf{N}_{\psi(1)}$ and $\mathbf{N}_{\psi(2)}$ are polarizations normalized to the intensity of the waves $\psi^{(1)}$ and $\psi^{(2)}$ respectively. Figure $a$ ) shows the situation then the intensities of $\psi^{(1)}$ and $\psi^{(2)}$ are equal; $b$ ) then the intensity of $\psi^{(2)}$ is greater than $\left.\psi^{(1)} ; c\right)$ then the intensity of $\psi^{(2)}$ is less than $\psi^{(1)}$.

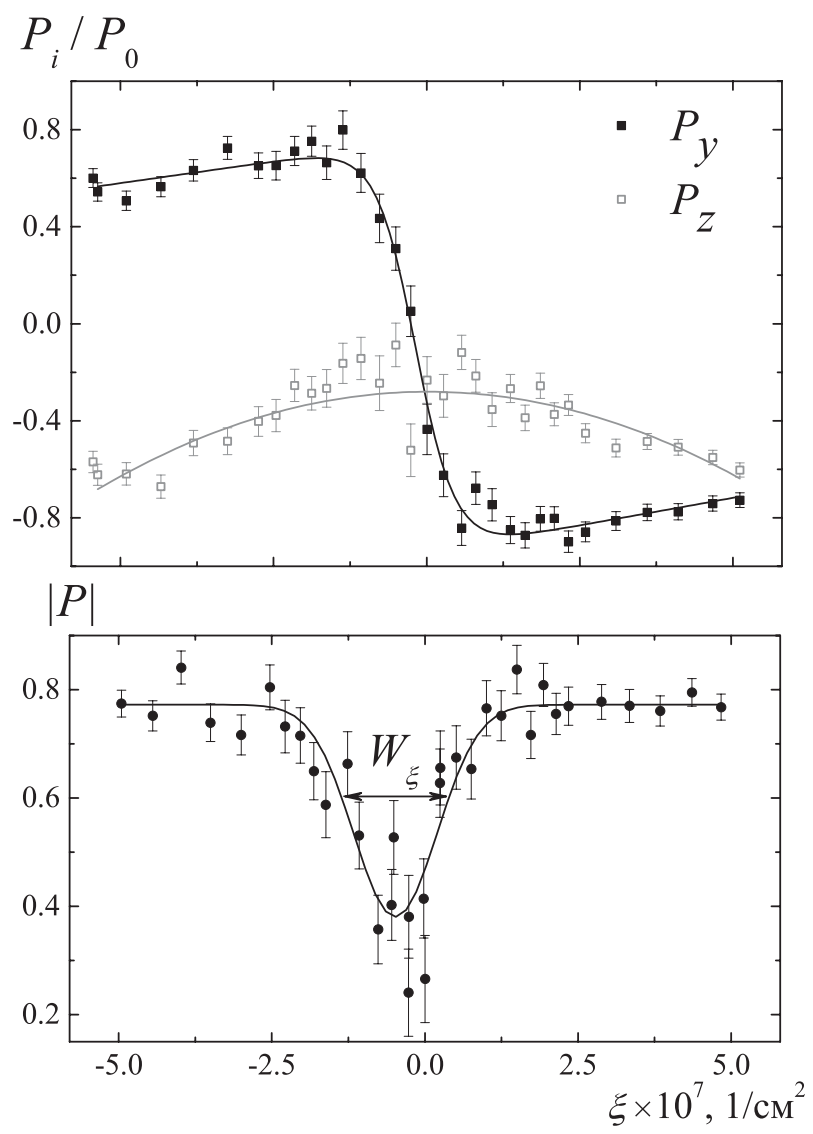

Figure 7. Polarization of the diffracted beam as a function of the value of the crystal deformation $\xi$ for the Bragg angle $\theta_{\mathrm{B}}=82^{\circ}$. The top picture is the polarization components $P_{y}$ and $P_{z}$, the bottom picture is the absolute polarization value $|P|$. Solid lines are the fitting of experimental data.

\section{Experimental results}

Figure 7 shows the dependence of the polarization of the diffracted beam on the quadratic strain parameter $\xi$ of the crystal.

It is noteworthy that the entire range of the deformation parameters (see Fig. 7) corresponds to the temperature 


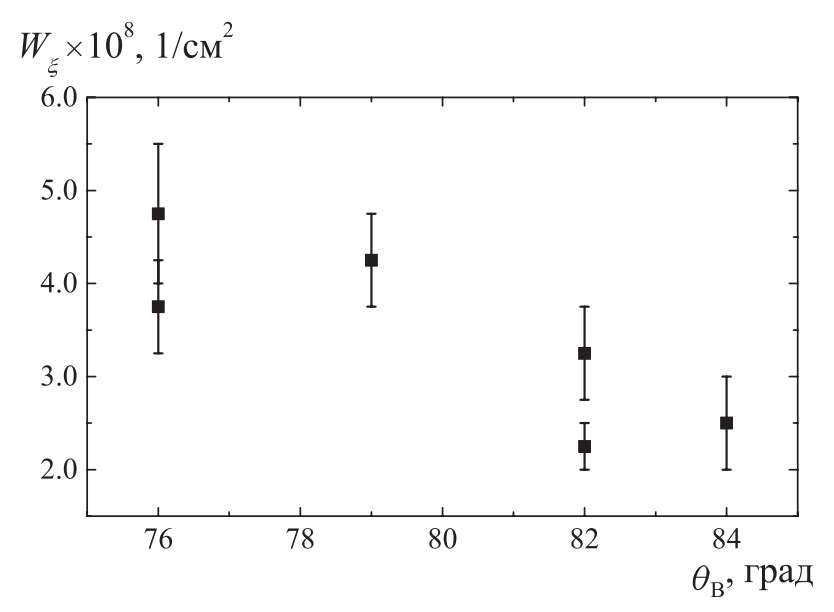

Figure 8. Dependence line width $W_{\xi}$, see Fig. 7, on the Bragg angle $\theta_{\mathrm{B}}$.

difference $\Delta T \approx \pm 2 \mathrm{~K}$ between the center of the crystal and its ends. Thus, already a small strain of the crystal corresponding to $\Delta T \approx 0.5 \mathrm{~K}$ per the entire crystal length of $14 \mathrm{~cm}$ leads to a change in the sign of the final polarization of the beam, i.e., to the focusing of one Bloch wave at the center and to the complete defocusing of the second Bloch wave; as a result, the latter wave leaves the crystal through its ends and is stopped by absorbers. Furthermore, it is seen that the depolarization of the beam is significant in a very narrow strain range(the line width in Fig. 7, bottom picture). Residual polarization in the absence of deformation is due to the inaccuracy of spin rotation by the angle $\pm \pi / 2$, because waves propagating in the crystal do not necessarily satisfy the exact Bragg condition.

Figure 8 shows the dependence of the strain range (line widths $W_{\xi}$ ), where the beam is depolarized, i.e., the intensities of two Bloch waves are comparable, on the angle of diffraction $\theta_{\mathrm{B}}$. As expected, the depolarization line width decreases with increasing Bragg angle.

\section{Conclusion}

In this work, the effect of neutron spin rotation at Laue diffraction in a weakly deformed transparent for the neutron noncentrosymmetric crystal has been studied experimentally for the first time. The effect is due to the Schwinger interaction of the magnetic moment of a neutron with the intracrystalline electric field depending on the degree and character of deformation of the crystal.

A method has been developed for controlled deforming a perfect single crystal by means of creating a temperature gradient in it. It has been shown that the polarization of diffracted neutron beams (direct and reflected) can be effectively controlled (e.g., its sign can be reversed at a certain thickness of the crystal) by slightly varying the strain (temperature gradient) of the crystal. Thereby, it has been shown that crystalline electric fields acting on a neutron in crystals with no center of symmetry can be determined from the measurements of the spin component perpendicular to the initial polarization of the beam. In addition, the possibility of controlling these fields in experiments on studying the fundamental properties of the neutron has been demonstrated.

We are grateful to the staff of the WWR-M reactor (Petersburg Nuclear Physics Institute, Gatchina, Russia). This work was supported by the Ministry of Education and Science of the Russian Federation (state contract no. 3.3838.2017/4.6).

\section{References}

[1] P.B. Hirsch, A. Howie, R.B. Nicholson et al., Electron microscopy of thin crystals (Butterworths, London, 1965)

[2] V.L. Alexeev, E.G. Lapin, E.K. Leushkin et al., Sov. Phys. JETP 67, 1727 (1988)

[3] V.L. Alexeev, V.V. Voronin, E.G. Lapin et al., JETP 69, 1083 (1989)

[4] V.L. Alexeev, V.V. Fedorov, E.G. Lapin et al., Nucl. Instr. Meth. A 284, 181 (1989)

[5] V.V. Voronin, E.G. Lapin, S.Y. Semenikhin et al., JETP Lett. 72, 308 (2000)

[6] V.V. Fedorov, E.G. Lapin, S.Y. Semenikhin et al., Physica B 297, 293 (2001)

[7] V.V. Fedorov, V.V. Voronin, E.G. Lapin et al., Tech. Phys. Lett. 21, 884 (1995)

[8] V.G. Baryshevskii, S.V. Cherepitsa, Phys. Status Solidi B 128, 379 (1985)

[9] V.V. Fedorov, E.G. Lapin, S.Y. Semenikhin et al., Int. J. Mod. Phys. A 23, 1435 (2008)

[10] V.V. Fedorov, M. Jentschel, I.A. Kuznetsov et al., Nuclear Physics A 827, 538c (2009)

[11] V.V. Voronin, V.V. Fedorov, S.Yu. Semenikhin et al., JETP Lett. 106, 481 (2017)

[12] N. Kato, J. Phys. Soc. Japan 19, 971 (1964) 